\title{
Evaluation of monitoring methods for electron beam melting powder bed fusion additive manufacturing technology
}

\author{
Paola M. Cordero ${ }^{1} \cdot$ Jorge Mireles $^{1}\left[0 \cdot\right.$ Shakerur Ridwan $^{1} \cdot$ Ryan B. Wicker $^{1}$
}

Received: 28 August 2015/Accepted: 13 November 2016/Published online: 1 December 2016

(C) Springer International Publishing Switzerland 2016

\begin{abstract}
In-process process sensing and monitoring is being incorporated across additive manufacturing technologies due to the need for part qualification. Implementation of additively manufactured end-use parts has been hindered by the inherent process variability and anisotropy that adversely affect part performance. Process monitoring methods have the potential to ensure fabrication integrity is achieved and part isotropy is maintained across the entirety of a part. This manuscript compares two methods (pyrometer and infrared thermography) that have the potential to monitor layerwise surfaces of a powder bed fusion process. Measurement of surface temperatures during fabrication can be useful for parameter development of novel materials, prediction of resulting microstructural architectures, and ultimately as feedback used in a closed-loop control system to allow full spatial and temporal control of melting and microstructure. A multi-wavelength pyrometer was externally mounted atop an electron beam melting (EBM) additive manufacturing system to observe and record surface temperatures during the fabrication process. The multiwavelength pyrometer used in this study was a non-contact device that was emissivity independent and capable of taking fixed spot sized measurements. An infrared camera was also installed atop an EBM system to monitor the fabrication surface and was used to measure temperature variations across the entire build area. Although the IR camera produces spatial measurements of an entire part, temperature data is emissivity dependent. Parts with variations in
\end{abstract}

Jorge Mireles

jmireles3@utep.edu

1 W.M. Keck Center for 3D Innovation, The University of Texas at El Paso, 500 W. University Ave., El Paso, TX 79968, USA processing were fabricated and monitored using each instrument. Thermal variations between parts were identified with each instrument and related to microstructure. The advantages and disadvantages of each monitoring device were documented and are discussed in this manuscript.

Keywords Additive manufacturing - Thermography · Pyrometry $\cdot$ Process monitoring $\cdot$ Powder bed fusion

\section{Introduction}

Additive manufacturing (AM) is a process that consists of adding layers of a material to create a solid 3D object starting from a computer-aided-design (CAD) [1]. Although there are various classes of AM technologies, this manuscript will focus on a powder bed fusion technology referred to as electron beam melting (EBM). The EBM process is an AM technology that has been used to fabricate end-use metal components in industries ranging from aerospace, biomedical, electrical, and automotive [2]. Fabrication of materials using EBM technology is of interest because it can inherently produce dense parts with reduced residual stresses compared to similar powder fusion processes like selective laser melting, thus, depending on their application, parts can be eventually utilized in their as-fabricated state. Today, the material processing in EBM is fully controlled by the machine and little feedback of processing is given to the user. In commercial EBM systems, temperature feedback consists of a single thermocouple that measures the temperature at a single point below the build platform. However, since powder bed fusion technology is a layer-by-layer process, the temperature readings are not representative of the layer being fabricated and do not show the surface temperatures 
to perceive any effect on the quality of the part (e.g., microstructure variations from different thermal conditions). When developing processing parameters or attempting to control a material's microstructure, it is important to know the surface temperature during fabrication. The added capability of knowing the temperature for each process step can allow the operator to modify system parameters as a means to obtain a desired surface temperature to achieve a tailored microstructure, enable qualification, and provide a means for in situ monitoring.

Previous researchers have developed various methods to understand the temperature variations of layerwise surfaces to analyze the thermal environment of the EBM system during processing and how it ultimately affects part quality. In a study conducted by Price et al. [3], an infrared (IR) camera was used in an EBM system to analyze the melt pool sizes and temperature distributions around the melting area at various configurations, finding peak temperatures during the melting step, molten pool emissivity, and small differences in temperature profiles as the build increases in height. Additionally, Rodriguez [4] recorded thermal images to obtain the post-melting temperature of the forming layer by utilizing an IR camera with a specified emissivity value for EBM while processing Ti-6Al-4V. Utilization of an IR camera in EBM has allowed analysis of thermal images to detect defects such as porosity and the development of automated control for microstructure and process control [5]. The studies performed by Rodriguez and Mireles utilized a shutter mechanism to avoid metallization of the viewing window, due to elements condensing and evaporating from the melt pool. Oak Ridge National Laboratory developed a shutterless mechanism to obtain realtime thermal images using a moving Mylar film canister to improve the feedback of the EBM process and found that continuous data could be acquired that is representative of various process steps, including preheating, melting, and powder deposition [6].

The aforementioned monitoring methods focused on the use of infrared emissions as a source of thermal information; however, a variety of factors such as radiant temperature and emissivity can affect the thermal measurement. In this study, a monitoring method was used for an IR camera while ensuring consideration of emissivity and mean radiant temperature for the melt surface of an EBM-fabricated part. The thermal variations due to emissivity are identified in this manuscript. For comparison, a multi-wavelength pyrometer was also used, which was a device that calculates the surface temperature of a target object without the need of knowing emissivity. The multi-wavelength pyrometer was also externally mounted atop an EBM system to monitor the surface temperature during the fabrication process. Temperature data from the pyrometer was compared to that of a thermocouple. The pyrometer allowed the characterization of the EBM process, such as powder deposition, preheating, melting, and cooling during layer-by-layer fabrication. Both instruments were used to monitor the fabrication of cylinders under different processing conditions. It was found that both instruments were capable of identifying thermal variations resulting from process changes that were later found to affect part microstructure. Thermal information using either monitoring device can be useful when developing and improving parameters for nonstandard materials or to improve a part's mechanical properties by further controlling the thermal environment during fabrication. Furthermore, the temperatures profiles obtained can give an approximation of the solidification phenomena that occurs during each layer, which may be useful to achieve full spatial and temporal control of microstructure.

\section{Methodology}

\subsection{EBM system}

An Arcam S12 EBM system from ARCAM AB (Mölndal, Sweden) was used in this study to fabricate parts using Ti$6 \mathrm{Al}-4 \mathrm{~V}$ powder precursor material. The EBM process consists of several steps to fabricate a part that include powder deposition, preheating, and melting. First, a layer ( $\sim 0.07 \mathrm{~mm}$ in thickness) of powder is deposited into the build platform by a powder deposition mechanism. The electron beam preheats the powder to about $50 \%$ of the melting temperature of the alloy $\left(\sim 760^{\circ} \mathrm{C}\right.$ for Ti-6Al-4V) that is used to sinter the powder around the part to hold the part during the fabrication process [7]. Furthermore, preheating helps decrease the thermal gradient between layers and throughout the overall part, thus, reducing thermal stresses [7]. After the preheat cycle, the electron beam melts the metal powder following the $2 \mathrm{D}$ cross section from a sliced computer-aided design (CAD) model. The build platform is lowered by one layer thickness and powder deposition, preheating, and melting are repeated until fabrication is complete [7].

\subsection{Multi-wavelength pyrometer}

The pyrometer specified in this study has been previously used to measure processing temperature of metals in hostile thermal environments where the emissivity changes or is unknown, such as investment casting. The multi-wavelength pyrometer has been able to identify and discard radiation affected by the process's gas emissions or absorptions while errors due to optics transmission are accounted for upon instrument calibration [8]. Furthermore, the pyrometer is a non-contact device capable of measuring the temperature of an object without the need of knowing emissivity, or an object's surface ability to emit 
radiant energy [9]. The measurement device is capable of taking up to seven measurements per second. To automatically determine an emissivity, the instrument measures the target object's wavelengths to calculate a temperature whose radiance curve is compared to an ideal Planck curve. If the curve matches, the target is said to be an ideal blackbody (emissivity $=1$ ), otherwise the radiance corresponds to a non-blackbody and emissivity is automatically calculated to match a Planck curve.

All experiments performed using the multi-wavelength pyrometer consisted of pointing the pyrometer to the middle of the build platform, thus, all recorded temperature data corresponded to the part fabricated at that location. In this research, the multi-wavelength pyrometer was externally mounted atop an EBM system pointing to the build platform within the vacuum chamber looking through a quartz window (which allowed $\sim 99 \%$ transmission for detected wavelengths) (Fig. 1a). Figure 1 illustrates the position of the pyrometer where the spot within the build platform is the measurement area ( $\sim 2 \mathrm{~mm}$ diameter) of the pyrometer (Fig. 1b). The multiwavelength pyrometer functions using a fiber optic cable that directs light to the object whose temperature is being measured and detects the wavelengths emitted using a spectrophotometer that separates the detected wavelengths. By employing an electrical transmission line, the information is sent to the device's analog-to-digital conversion system. The multi-wavelength pyrometer is able to measure temperatures in the range of $300-2000{ }^{\circ} \mathrm{C}$. While measuring, the pyrometer displays and records (1) temperature of the target object (in ${ }^{\circ} \mathrm{C}$ ), (2) tolerance of the measured temperature (standard deviation), and (3) signal strength (an emissivity value at a certain wavelength) [9]. Figure 2 represents the experimental setup that shows the pyrometer mounted atop the EBM system with extra hardware surrounding the installation to prevent radiation exposure to the user.

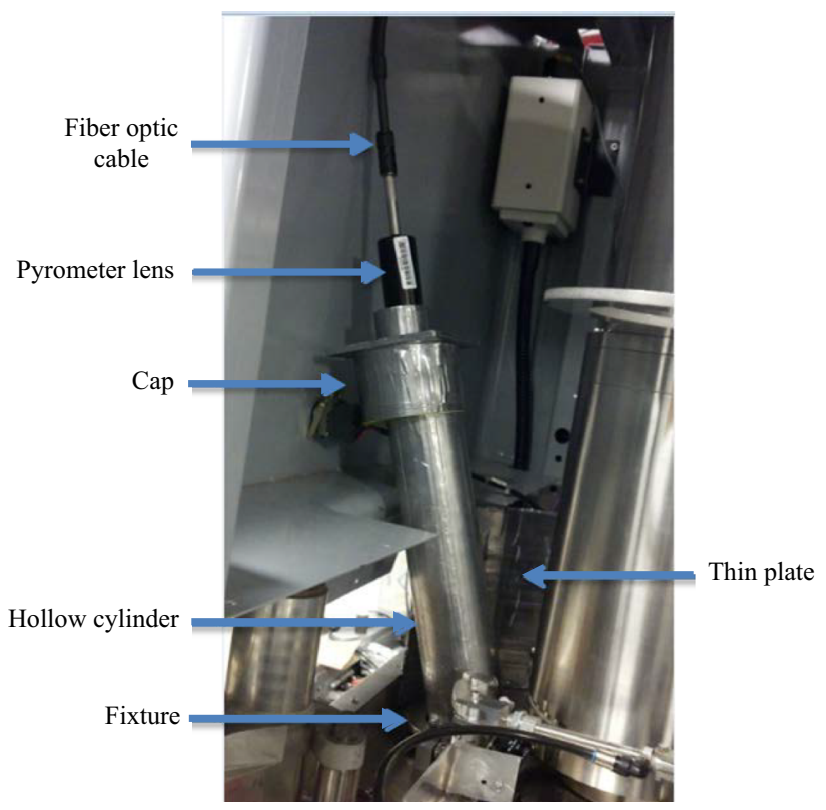

Fig. 2 Experimental setup for the multi-wavelength pyrometer in the EBM system

\subsection{Infrared thermography}

An SC645 FLIR IR camera was utilized in this study, which is capable to taking up to twenty-five measurements per second. Five holes were drilled atop the EBM vacuum chamber to accommodate fixtures used to mount an IR camera. All the holes were drilled with precaution to preserve the high vacuum requirement necessary for the fabrication process. A frame was installed to hold the IR camera atop the machine build chamber. A ZnSe glass was used for its high transmittance of IR wavelengths. A pneumatic controlled shutter mechanism was installed to prevent the ZnSe glass from metallizing, which occurs within the fabrication environment when elements are vaporized and coated onto inner surfaces during melting.

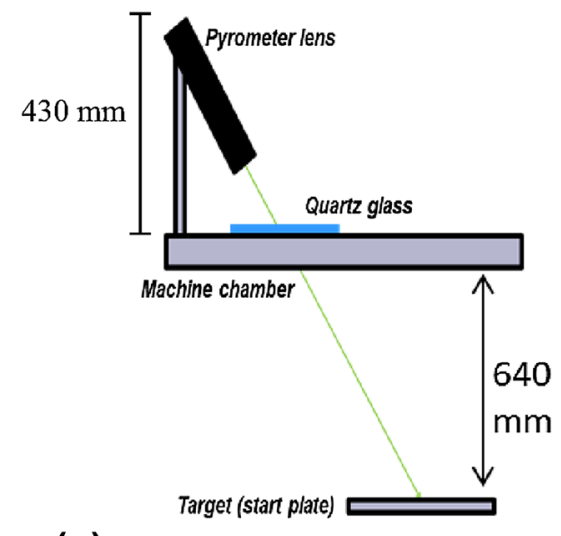

(a) Pyrometer on the EBM system

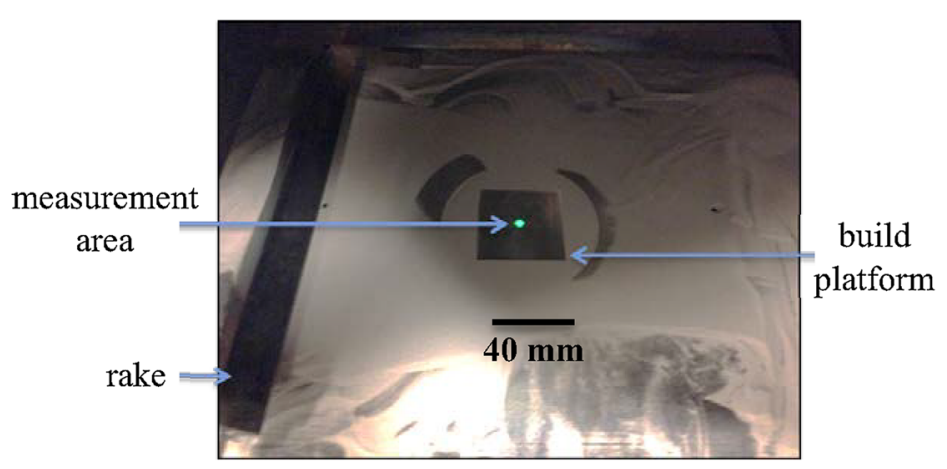

(b) Start plate and pyrometer setup

Fig. 1 a Pyrometer setup schematic, b laser spot specifying area where pyrometer obtained data 


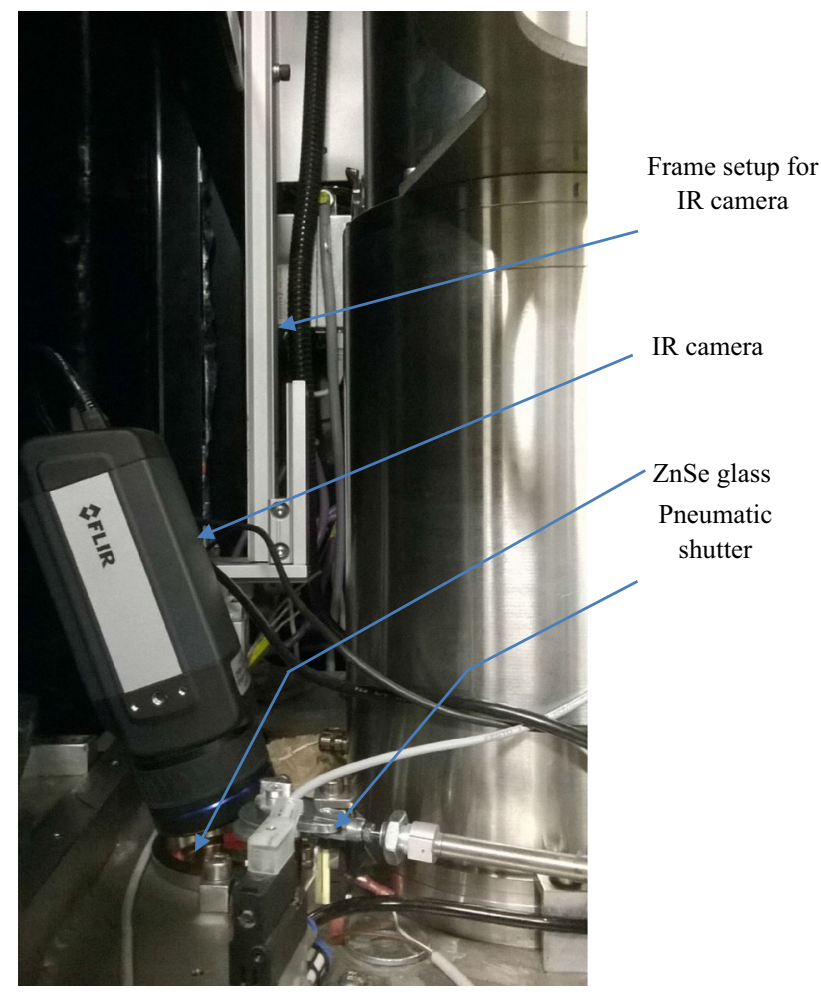

Fig. 3 Experimental setup of ARCAM A2 machine upgraded with IR Camera

The shutter pneumatic system was controlled through an electromechanical switch (relay) and the relay was controlled using LabVIEW. An NI Compact-RIO (National Instruments, Austin, TX) was used to control the relay mechanism. Air-supply was connected to the input regulator on the pneumatic panel coupled with a pneumatic piston to actuate the shutter. Based on the state of relay switch the shutter was opened or closed. The logic state of the relay was controlled using LabVIEW through the Compact-RIO that was correlated with the signal received from the machine. One of the modules of the Compact-RIO received signals from the machine to determine the on/off state of the electron beam. Figure 3 shows the experimental setup used to affix the camera, as well as the $\mathrm{ZnSe}$ glass and shutter mechanism installed atop the EBM system with the aforementioned hardware additions.

\subsection{Description of experiments}

Of interest was obtaining temperature measurements from the multi-wavelength pyrometer and infrared camera installed atop the EBM system during fabrication of Ti6Al-4V cylinders measuring $1.5 \mathrm{~cm}$ in diameter and $2 \mathrm{~cm}$ in length. The cylinders were built with varying beam scanning parameters to record temperature versus time data. The melt scan rates for this study included 1000 and
$100 \mathrm{~mm} \mathrm{~s}^{-1}$. Part fabrication started with a CAD design that was converted into an STL format for use with the EBM system. Each cylinder was fabricated separately. The parts were centered at the start plate with a build direction parallel to the $Z$-axis of the building platform. The start plate was marked with an " $X$ " signaling the center of the part and beam calibration was performed in reference to the center of the ' $X$ '. Specific to the pyrometer, an extra calibration step required aligning the measurement spot to the center for the marked ' $\mathrm{X}$ '. Four parts were fabricated and labeled as 'Group A' and 'Group B'. Group A was fabricated using the standard recipe parameters for Ti-6Al-4V as specified by the system manufacturer, while Group B was fabricated with alternate parameters (10 times reduced beam speed) certifying a reduction in scan melt rate and each group contained at least one cylinder for each monitoring instrument. Layer-by-layer surface temperature monitoring data was obtained using each instrument for scan melt rates of $1000 \mathrm{~mm} \mathrm{~s}^{-1}$ for Group A and $100 \mathrm{~mm} \mathrm{~s}^{-1}$ for Group B.

\subsection{Microstructure}

The area of interest for microstructural analysis was the top surface of the cylinders, which was the area perpendicular to build direction and the pyrometer measurement area. The cylinders were cleaned in an ultrasonic bath for $15 \mathrm{~min}$ prior to metallographic preparation. The parts were mounted using Koldmount acrylic resin powder (Nobilium, Albany, New York) and curing resin in a 2:1 ratio, followed by grinding up to 1200 grit silicon carbide paper, polishing with alumina slurries and etching using Kroll's reagent consisting of $100 \mathrm{~mL}$ water, $2.5 \mathrm{~mL}$ hydrofluoric acid, and $5 \mathrm{~mL}$ nitric acid for 3-5 s. Once the microstructure was revealed, optical images were obtained using a Reichert MEF4 A/M with an imaging system to characterize grain morphology corresponding to each fabrication parameter.

\section{Results}

\subsection{Multi-wavelength pyrometer}

Surface temperature measurements were obtained from the multi-wavelength pyrometer for the different beam scan speeds and are illustrated in Fig. 4 where the red plot is the thermocouple data (temperature under the build platform), and the blue plot is data obtained from the multi-wavelength pyrometer (Fig. 4a represents the $1000 \mathrm{~mm} \mathrm{~s}^{-1}$ and Fig. $4 \mathrm{~b}$ represents the $100 \mathrm{~mm} \mathrm{~s}^{-1}$ scan speed). Zoomed in views of Fig. 4 (areas within boxes in the plots) are illustrated in Fig. 5 for each beam scan speed which defines 
Fig. 4 Layer by layer thermal data for Ti-6Al-4V EBM processing using multi-wave pyrometer for scan melt rates of; a $1000 \mathrm{~mm} \mathrm{~s}^{-1}$ and

b $100 \mathrm{~mm} \mathrm{~s}^{-1}$ where embedded boxes represent areas zoomed in for Fig. 4
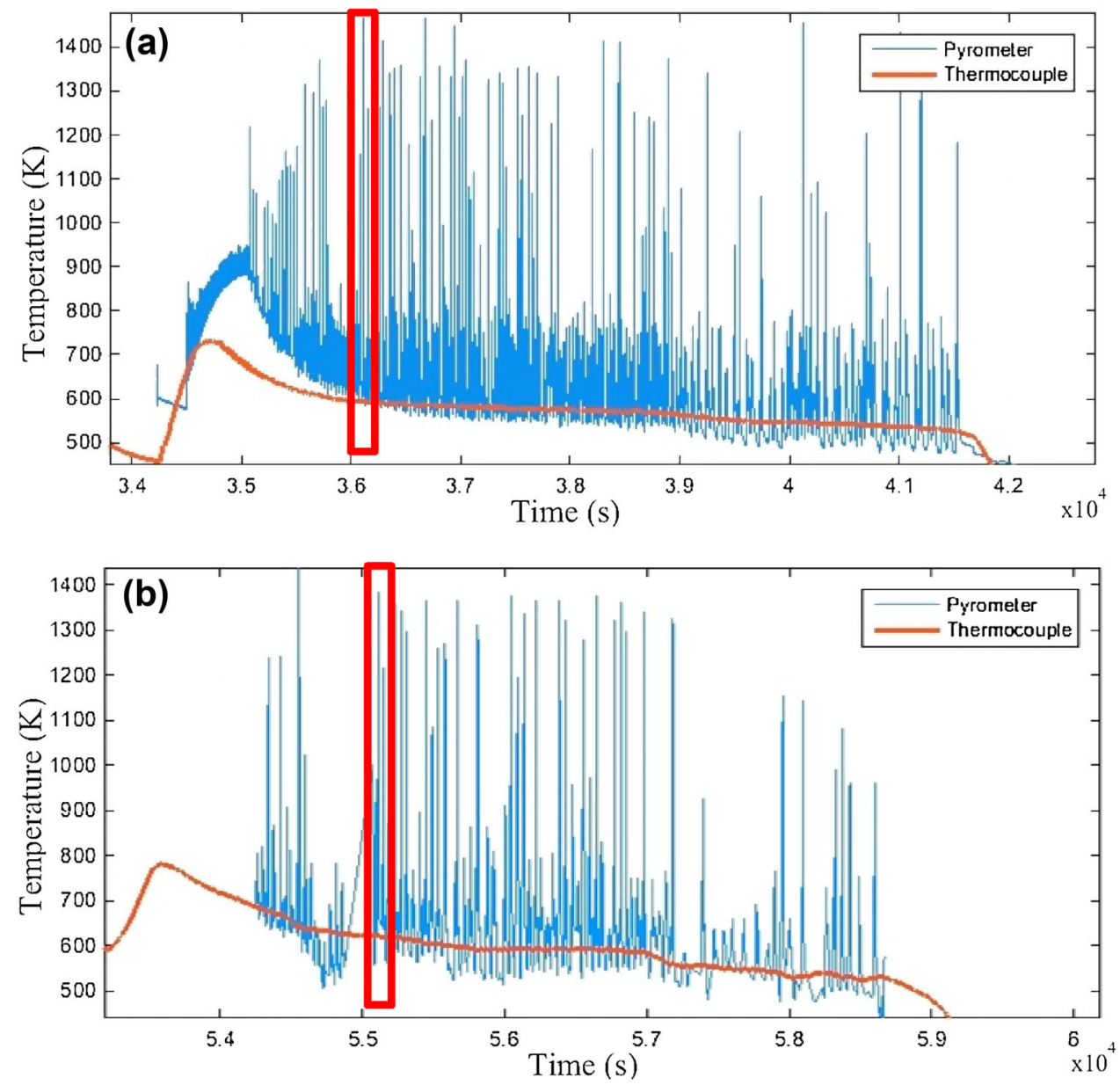
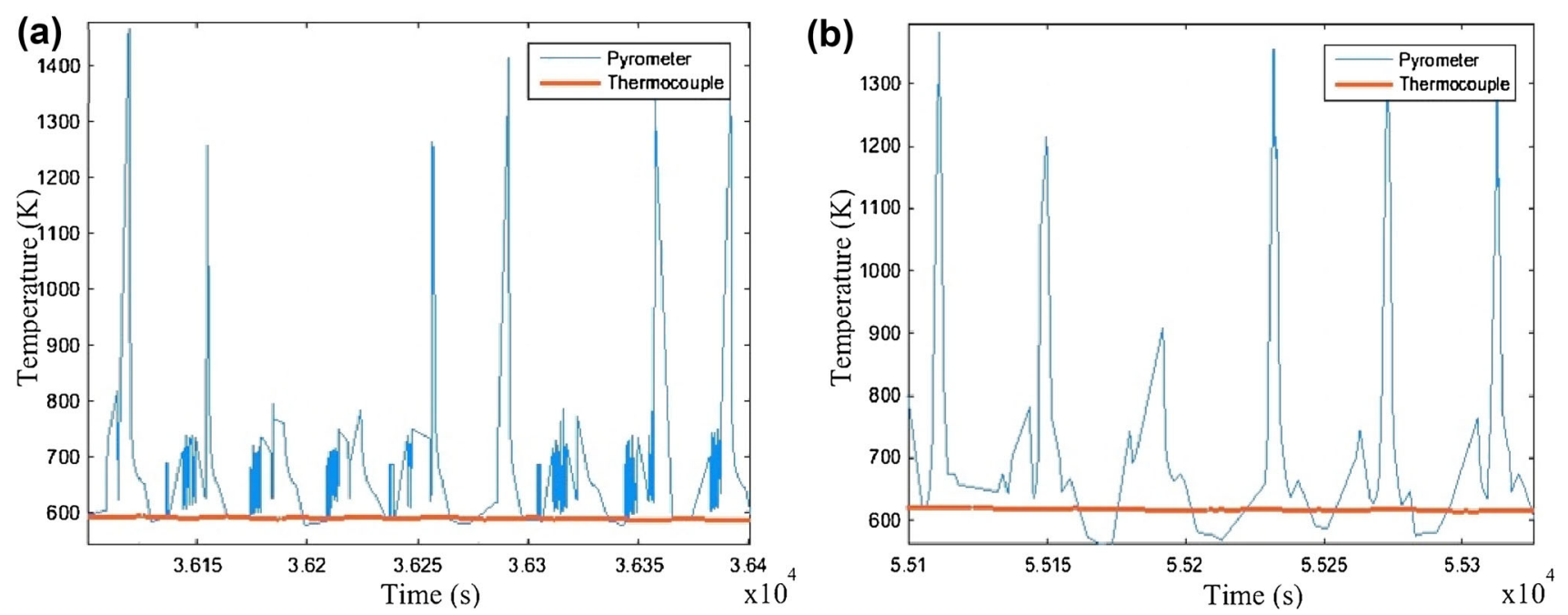

Fig. 5 Characterization of data for Ti-6Al-4V EBM processing using multi-wave pyrometer for scan melt rates of; a $1000 \mathrm{~mm} \mathrm{~s}^{-1}$ and b $100 \mathrm{~mm} \mathrm{~s}^{-1}$

each EBM process step more closely. Figure 5a, b show peaks that represent the melting step of the EBM process preceded by a preheating step and proceeded by a powder deposition step. It is important to note that Group B showed discrepancies owing to pyrometer position shifting from the center toward the end of fabrication (illustrated in Fig. 5b). Errors may also occur due to metallization of the viewing glass, which degrade the transmission of 
electromagnetic radiation. Revolving films or shutters can help ensure metallization is mitigated throughout fabrication. Discrepancies due to the movement of the measurement spot away from the fabrication sample is a disadvantage of this monitoring device since it can only measure a single spot around the fabricated area. Advanced fixtures may help ensure a spot is maintained within a specific area; however, space to allow monitoring is limited to a $70 \mathrm{~mm}$ viewport.

It is important to note that the graphs in Fig. 5 whose peaks depict the melt cycle of Ti-6Al-4V (melting point of $\sim 1350-1400{ }^{\circ} \mathrm{C}$ at the vacuum pressure of $\sim 10^{-3}$ Torr) sometimes do not reach these expected values. Thus, when analyzing data from a pyrometer, it is important to further analyze raw data that represents the strength of thermal radiation as a function of wavelength. Figure 6 shows raw data from the pyrometer that has been corrected for instrument response, which depicts the spectral radiance versus wavelength curve for a representative melt curve of the EBM-fabrication process. When looking at raw data, the following form of Planck's radiation formula can be used to calculate the temperature of each melt point:

$T=\frac{C\left(\frac{1}{\lambda_{1}}-\frac{1}{\lambda_{2}}\right)}{\ln \left(\frac{I_{1}}{I_{2}}\right)-5 \ln \left(\frac{\lambda_{2}}{\lambda_{1}}\right)}$

where $T$ is the temperature, $C$ is the second radiation constant, $\lambda_{1}$ and $\lambda_{2}$ are wavelengths selected from spectral data, and $\mathbf{I}_{1}$ and $\mathbf{I}_{2}$ are the ratio intensities corresponding to the selected wavelengths. It should be noted that Planck's equation yields higher precision when the selected wavelengths are further apart, as shown by experimentation provided by Felice in 1998. Although the data in Fig. 5 suggests the melting point is not achieved during a melt step, extracting raw data and calculating a temperature using Planck's radiation formula yields a result that coincides with the melt step. A total of ten different melt peaks were analyzed and it was found that the average melt step temperature for Group A was $1429 \pm 32{ }^{\circ} \mathrm{C}$. A similar approach was done with raw data from Group B where it was determined that the average melt step temperature was $1375 \pm 21{ }^{\circ} \mathrm{C}$. The most important observation of data obtained from Group B was the time that the part was maintained above $1000{ }^{\circ} \mathrm{C}$ during the melt step. The time that the parts in Group A were maintained above $1000{ }^{\circ} \mathrm{C}$ was $\sim 0.3 \mathrm{~s}$, while the parts from Group B were maintained above $1000{ }^{\circ} \mathrm{C}$ for $\sim 1.5 \mathrm{~s}$. This phenomenon can be observed from the graphs in Fig. 5 where the peaks are noticeably wider for Group B than Group A. Later, the effects of these temperature variations on the part's microstructure will be discussed which will lead into the conclusion that if the pyrometer's data is correctly analyzed, resulting data can be used to obtain an indication of part quality.

\subsection{Infrared thermography}

Figures 7 and 8 show the temperature profile of a part fabricated with standard parameters (scan speed $\sim 1000 \mathrm{~mm} / \mathrm{s}$ ) and manipulated parameters of slower scan speed $(100 \mathrm{~m} / \mathrm{s})$. Figure $7 \mathrm{a}-\mathrm{c}$ represent the cooling curve for the build height of 1,5 and $8.75 \mathrm{~mm}$, respectively. Figure $6 \mathrm{~d}$ represents a plot of temperature data for several layers of the fabricated part. The data were acquired using ThermaCAM software, which calculates the temperature based on the radiation captured and the emissivity of the object which have been defined as constants based on previous work by Rodriguez (2013). Each peak in Fig. 6d represents the start of melting. Since the emissivity of the powder is not same as the emissivity of the molten pool, the temperature data before each melting peak is not an accurate temperature value. That is, the graph shows areas where the temperature value is higher than the temperature of the formed object because the emissivity of the deposited power is higher $(0.5)$ than the emissivity of the formed object (0.26) (Rodriguez 2013). Nonetheless, the emissivity of the object after the melt step (0.26) was used for all data analyzed using ThremaCAM.
Fig. 6 Spectral radiance versus wavelength curve for a representative melt curve of the EBM-fabrication process

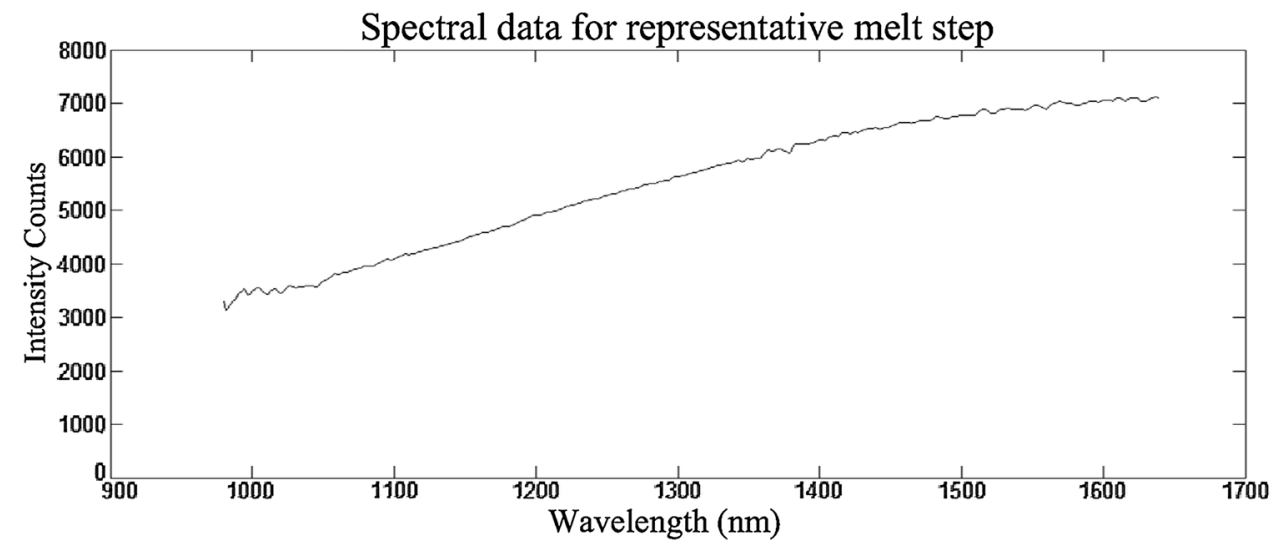



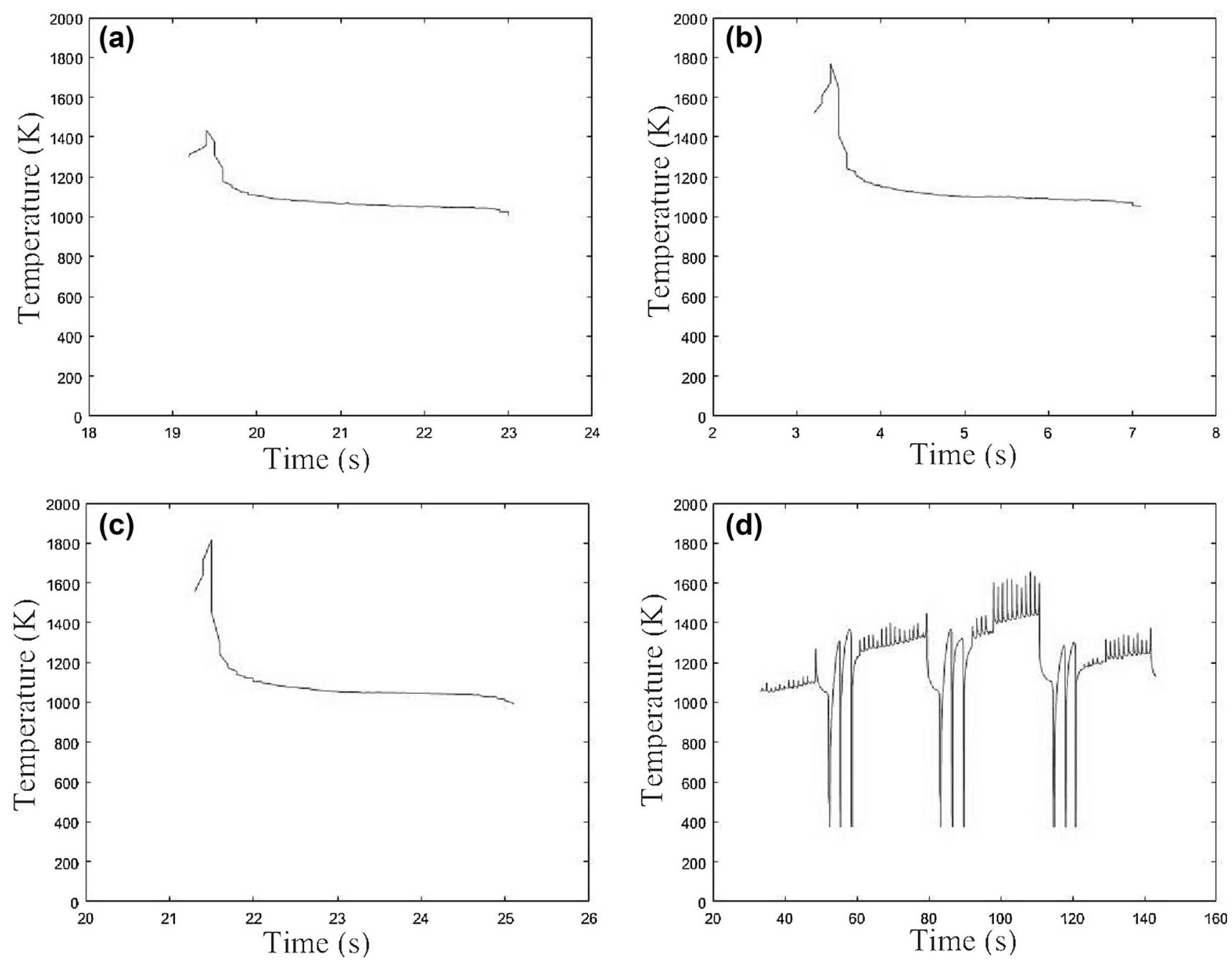

Fig. 7 Temperature profile of single layer fabrication at height $\sim 1 \mathrm{~mm} \mathbf{a}, \sim 5 \mathrm{~mm} \mathbf{b}$ and $\sim 8.75 \mathrm{~mm} \mathbf{c}$ and temperature profile of several layers of fabrication at $\sim 1 \mathrm{~mm}$ at the point of analysis using ARCAM build parameters (scan speed $1000 \mathrm{~mm} \mathrm{~s}^{-1}$ )

The change of melting temperature was observed for different adjacent layers. Figure $7 \mathrm{a}-\mathrm{c}$ shows that, for each height of fabrication, temperature of the molten pool decays almost up to $\sim 1100 \mathrm{~K}$. However, for the height of $8.75 \mathrm{~mm}$, the molten pool cools down more rapidly when compared to the height of 5 and $1 \mathrm{~mm}$, which can be noted by the steepness of the curve in Fig. 7c when compared to Fig. 7a, b. Thus, it can be concluded that the higher the build height, the higher the temperature difference to cool down which is most likely due to the high starting temperature. As previously discussed, various factors can affect the temperature measurement obtained from IR thermography. It is assumed that the measures taken in this study to approximate the radiation effects, emissivity, and optical transmission, give a close approximation of real temperature values that are representative of the fabrication process.

For the cylinder fabricated with a reduced scan speed of $100 \mathrm{~mm} / \mathrm{s}$, the temperature profile was found to be different for a comparable measurement area to the one selected in Fig. 7 (approximately at the center of the cylinder). Figure $8 \mathrm{a}-\mathrm{c}$ represents the cooling curve at the height of $\sim 1,5$, and $8.75 \mathrm{~mm}$, respectively, while Fig. $8 \mathrm{~d}$ represents the curve for several layers. For a single layer, a fluctuating temperature profile was obtained in case of each height instead of a smooth curve, as found in the curves corresponding to Fig. 7. The scan speed was one tenth of the regular build parameter, thus the molten pool, as well as the fabricated pool area render sufficient time for conduction across the fabricated sample. Since the scan of the beam for the next hatching direction was slow $\left(100 \mathrm{~mm} \mathrm{~s}^{-1}\right)$, heat transfers up to the specific point of analysis by the conduction process, which results in an increase in temperature. The same effect is seen from data taken by the pyrometer, where there is a prolonged period where a spot is being exposed to a higher temperature. Once again, although the melt peaks did not show an 

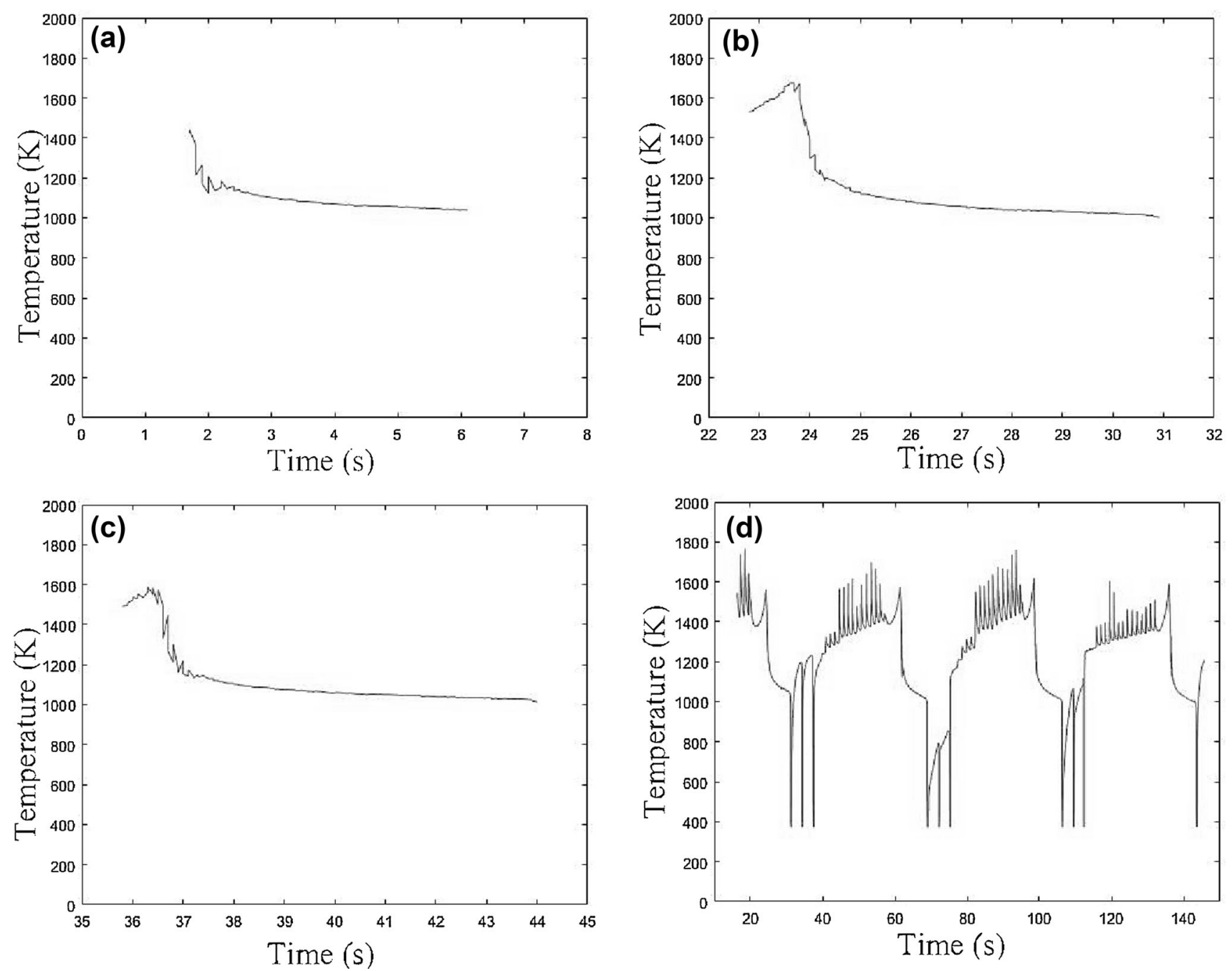

Fig. 8 Temperature profile of single layer fabrication at height $\sim 1 \mathrm{~mm} \mathbf{a}, \sim 5 \mathrm{~mm} \mathbf{b}$ and $\sim 8.75 \mathrm{~mm} \mathbf{c}$ and $\mathbf{d}$ temperature profile of several layers of fabrication at $\sim 1 \mathrm{~mm}$ at the point of analysis using scan speed $100 \mathrm{~mm} \mathrm{~s}^{-1}$ and beam current $2.5 \mathrm{~mA}$

appreciable change in maximum temperature, the cooling curves show different thermal behaviors that were later shown to affect microstructure. It is believed that faster scan rates provides less duration at the $\beta$ transus temperature and consequently less percent amount of $\beta$ phase would be formed.

\subsection{Microstructural characterization}

EBM-fabrications of Ti-6Al-4V exhibited a typical Widmanstätten microstructure consisting of discrete $\alpha$ phase in continuous $\beta$ phase. Figure 9 illustrates the microstructure variations in response to scan melt rates occurring at 1000 and $100 \mathrm{~mm} \mathrm{~s}^{-1}$. The varying scans yielded different thermal behaviors that would be found to affect the thickness of the $\alpha$-platelets. It can be noted that the $\alpha$-plate thickness decreases for the rapid melt scan rate, and that the microstructure for such is more acicular in contrast to the slower melt scan which exhibits a more lamellar like microstructure. The $\alpha$-plate thickness in Fig. 9a corresponds to an average of $0.86 \pm 0.20 \mu \mathrm{m}$ while an average $\alpha$-plate thickness of $1.23 \pm 0.19 \mu \mathrm{m}$ corresponds to Fig. 9b. Correlations between microstructures obtained during EBM-fabrication of Ti-6Al-4V parts and real-time temperatures recorded by a pyrometer for one build and by an infrared camera for a separate build were used to evaluate the effect of parameter modifications on microstructure. The variation in build parameters during fabrication of Group B contributed a greater energy input, which resulted in a higher chamber temperature at $709{ }^{\circ} \mathrm{C}$ (as shown by the thermocouple readings in Figs. 4, 5) and prolonged exposure to increased temperatures, while the fabrication of Group A took place at a chamber temperature of $650{ }^{\circ} \mathrm{C}$ (as shown by the thermocouple readings in Figs. 4, 5). Both the effects of build temperature and prolonged exposure to thermal energy due to the slower scan 

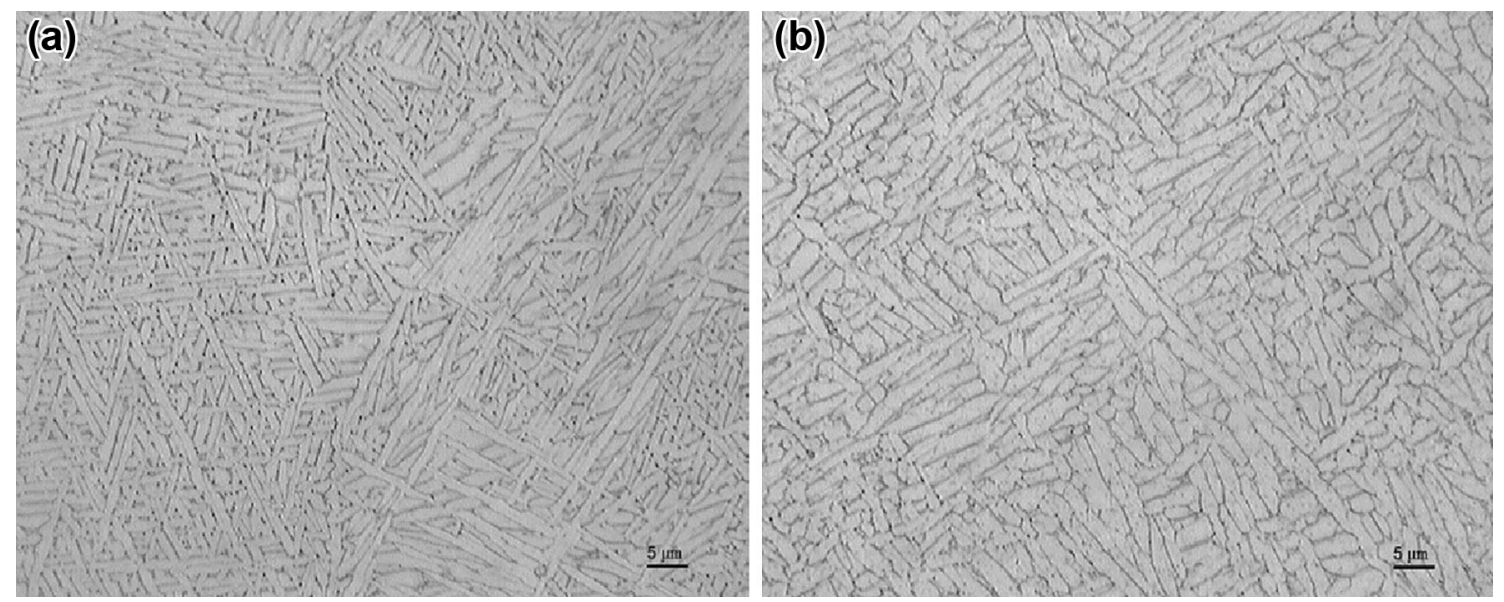

Fig. 9 Micrographs of $\alpha+\beta$ Widmanstätten Ti-6Al-4V parts fabricated by EBM at scan melt rates of $1000 \mathrm{~mm} \mathrm{~s}^{-1}$ (a) and $100 \mathrm{~mm} \mathrm{~s}^{-1}$ (b)

speed are expected to contribute to continuous grain growth. If the graphs for the different scan speeds are compared, it can be seen that the cooling rate for the faster scan speed is on the order of $2250 \mathrm{~K} \mathrm{~s}^{-1}$ while the slower scan speed has a cooling rate on the order of $1700 \mathrm{~K} \mathrm{~s}^{-1}$. Although research on cooling rates in EBM is limited, this work coincides with others who have identified cooling rates for EBM to be well in excess of $410 \mathrm{~K} \mathrm{~s}^{-1}$ [10]. The microstructure observed in Fig. 9 correlates closely to these data, as well as previous work by others [11] where it is shown that as cooling rate decreases, $\alpha$ lath thickness increases. The ability to successfully monitor the fabrication process in powder bed fusion additive manufacturing will aid in the analysis and understanding of guidelines pertaining to the effects of processing on part microstructure and can help further define if a process is within a desired specification. Furthermore measurement of surface temperatures during fabrication can be useful for parameter development of novel materials, prediction of resulting microstructural architectures, and ultimately as feedback used in a closed-loop control systems to allow full spatial and temporal control of melting and microstructure.

\section{Conclusions}

The most commonly used method for temperature feedback of powder bed fusion technology is a thermocouple; however, this measurement technique does not give a representation of actual fabrication results. In this study, two non-contact measurement techniques were evaluated to gain a better understanding of the fabrication process as it relates to the layer-by-layer forming process, which can eventually be used for part qualification. A multi-wavelength pyrometer was evaluated which was capable of recording process specific emissivity independent temperature data. Although the temperature data that is directly output by the instrument is helpful in identifying process temperatures, a further analysis of raw spectral data needed to be performed to analyze the thermal behavior more closely. Moreover, the pyrometer was only capable of measuring a fixed spot size that only represents thermal behavior. An IR camera was also used to monitor part fabrication. It was found that, although this manuscript focuses on temperature monitoring, the IR camera was capable of identifying fabrication anomalies related to defects such as porosity, warping, and delamination, as has been shown by previous research (Mireles et al. 2015). Temperature data obtained from the IR camera; however, is subjected to effects from radiation within the fabrication chamber, as well as emissivity changes resulting from physical changes of the material throughout processing. Groups of cylinders were fabricated with variations in fabrication parameters aimed at changing the thermal history of a fabricated part. It was found that both instruments were capable of capturing thermal differences resulting from process parameter modifications that were later found to affect microstructure. Although the instruments selected in this study were evaluated as separate entities, it may be ideal to implement both instruments together. In this case, it may be possible to add a witness coupon to a build file that the pyrometer is able to measure throughout the fabrication process of an end-use part. The measurement data obtained can then be used to verify that the melting point of the material is being achieved and also ensure that the measurements of both thermal sensors are within agreement with each other. Nonetheless, the use of either instrument can be very helpful in the growing area of part qualification and in-process process monitoring for not only EBM but other additive manufacturing technologies such as directed energy deposition methods and selective laser melting. 
Acknowledgements The research presented here was performed at The University of Texas at El Paso (UTEP) within the W.M. Keck Center for 3D Innovation, expanding recently to over 13,000 sq. ft. and providing access to state-of-the-art facilities and equipment as a result of funding, most recently, from the State of Texas Emerging Technology Fund. The authors are grateful to Jonathan Minjares, Philip Morton, Diego Mejorado and Israel Segura for their assistance on various aspects of the project. Support for this project was provided by the National Science Foundation through Grant 1405526.

\section{References}

1. Sclater N, Chironis NP (2006) Mechanisms and mechanical devices sourcebook. McGraw-Hill Professional, New York

2. Gibson I, Rosen DW, Stucker B (2010) Additive manufacturing technologies. Springer, New York

3. Price S, Lydon J, Cooper K, Chou K (2013) Experimental temperature analysis of powder-based electron beam additive manufacturing. In: Solid Freeform Fabrication Symposium Proceedings, pp 162-173

4. Rodriguez E (2013) Development of a thermal imaging feedback control system in electron beam melting. El Paso: ProQuest LLC, Thesis

5. Mireles J (2013) Process study and control of electron beam melting technology using infrared thermography. El Paso: PLC, Thesis
6. Dinwiddie RB et al (2013) Thermographic in situ process monitoring of the electron-beam melting technology used in additive manufacturing. In: SPIE defense, security, and sensing. International society for optics and photonics

7. Cormier D, Harrysson O, West H (2004) Characterization of H13 steel produced via electron beam melting. Rapid Prototyp J 10(1):35-41

8. Felice RA (2003) Expert system spectropyrometer results for non-black, non-grey, or changing emissivity and selectively absorbing environments. Electro-Techno-Exposition, Moscow

9. Felice RA (2006) Investment casting temperature measurement. Foundry Manag Technol 134(8):40-41

10. Bermani S, Blackmore ML, Zhang W, Todd I (2010) The origin of microstructural diversity, texture, and mechanical properties in electron beam melted Ti6Al4V. Metall Mater Trans A 41(13):3422-3434

11. Hrabe N, Quinn T (2013) Effects of processing on microstructure and mechanical properties of a titanium alloy (Ti-6Al-4V) fabricated using electron beam melting (EBM). Part 2: enegy input, orientation, and location. Mater Sci Eng A 573:271-277

12. Mireles J, Ridwan S, Morton P A, Hinojos A, Wicker R B (2015). Analysis and correction of defects within parts fabricated using powder bed fusion technology. Surf Topogr: Metrol Prop 3(3). doi:10.1088/2051-672X/3/3/034002 